\title{
Vers une écriture rhizomatique : style et syntaxe dans La Bataille de Pharsale de Claude Simon
}

\author{
Ilias Yocaris \\ CIRCPLES - EA 3159, Université de Nice \\ David Zemmour \\ Classes préparatoires aux grandes écoles, Lille
}

Ceux qui croient pouvoir vaincre les labyrinthes en fuyant leurs difficultés restent en dehors. Italo Calvino, "Le Défi au labyrinthe »

\section{Remarques introductives}

La Bataille de Pharsale de Claude Simon (1969) est un des Nouveaux Romans qui s'écartent le plus des canons syntaxiques hérités du roman «classique »: la simple lecture de l'incipit suffit pour faire comprendre au lecteur qu'il a affaire à un agencement scriptural sui generis, sur lequel le " découpage » phrastique privilégié par la grammaire traditionnelle n'a pratiquement aucune prise.

\begin{abstract}
(1) Jaune et puis noir temps d'un battement de paupières et puis jaune de nouveau : ailes déployées forme d'arbalète rapide entre le soleil et l'œil ténèbres un instant sur le visage comme un velours une main un instant ténèbres puis lumière ou plutôt remémoration (avertissement ?) rappel des ténèbres jaillissant de bas en haut à une foudroyante rapidité palpables c'est-à-dire successivement le menton la bouche le nez le front pouvant les sentir et même olfactivement leur odeur moisie de caveau de tombeau comme une poignée de terre noire entendant en même temps le bruit de soie déchirée l'air froissé ou peut-être pas entendu perçu rien qu'imaginé oiseau flèche fustigeant fouettant déjà disparue l'empennage vibrant les traits mortels s'entrecroisant dessinant une voûte chuintante comme dans ce tableau vu où ? combat naval entre Vénitiens et Génois sur une mer bleu-noir crêtelée épineuse et d'une galère à l'autre l'arche empennée bourdonnante dans le ciel obscur l'un d'eux pénétrant dans sa bouche ouverte au moment où il s'élançait en avant l'épée levée entraînant ses soldats le transperçant clouant le cri au fond de sa gorge $(B P, 9)$
\end{abstract}

Dans notre exposé, qui constitue le prolongement direct d'une série de travaux plus ou moins récents sur l'écriture simonienne (v. notamment Rannoux 1997, Zemmour 1998, 2008, Yocaris 2002, 2006a, 2006b ; cf. également Piat 2005), nous nous fixons quatre objectifs: (a) décrire les transgressions syntaxiques mises en relief dans La Bataille de Pharsale; (b) montrer que ces transgressions confèrent au texte une dimension « rhizomatique », puisque chacune de ses composantes se trouve reliée aux autres par un tissu de connexions mouvantes qui se font et se défont sans cesse ; (c) pointer l'isomorphisme que l'on voit apparaître dès lors entre ce dispositif syntaxique et le dispositif narratif qu'il sous-tend, les deux offrant l'image d'un espace réticulaire qui se reconfigure en permanence ; (d) replacer le projet d'écriture ainsi dégagé dans un contexte conceptuel et historique plus vaste, en le rattachant aux invariants thématiques et stylistiques qui caractérisent les œuvres de fiction postmodernes.

\section{Le concept de « rhizome »}

Qu'est-ce qu'un rhizome ? Emprunté à la botanique, le terme est utilisé par Gilles Deleuze et Félix Guattari dans Mille plateaux pour désigner un espace organisationnel abstrait qui est absolument irréductible à toute unification. À la différence de la racine, le rhizome ne saurait être pensé comme un 
«objet» unitaire qui se subdiviserait sans cesse en fonction d'un schéma arborescent: dans sa forme ramifiée, il se présente au contraire comme une tige souterraine garnie de racines adventives inextricablement enchevêtrées (cf. fig. 1). Partant de ces données phytobiologiques somme toute assez banales, Deleuze et Guattari élaborent un concept philosophique d'une redoutable complexité, en montrant qu'un espace « rhizomatique » doit posséder les propriétés suivantes : (i) chacun des points de cet espace se trouve virtuellement connecté à tous les autres (cf. Deleuze et Guattari 1980 : 31) ; (ii) les éléments ainsi mis en relation sont fondamentalement hétérogènes (ibid. : 14); (iii) ils se retrouvent tous sur le même plan, sans qu'il soit possible de les hiérarchiser (ibid. : 25-26) ; (iv) leur fonction, leur nature et leur position se modifient en même temps que les rapports qui les relient entre eux (ibid. : 26 et passim); (v) l'ensemble du dispositif forme un perpetuum mobile qui se transforme qualitativement au fur et à mesure qu'il se complexifie (ibid. : 15).

FIG. 1 AGENCEMENTS RHIZOMATIQUES ET SYSTEMIES RACINAIRES
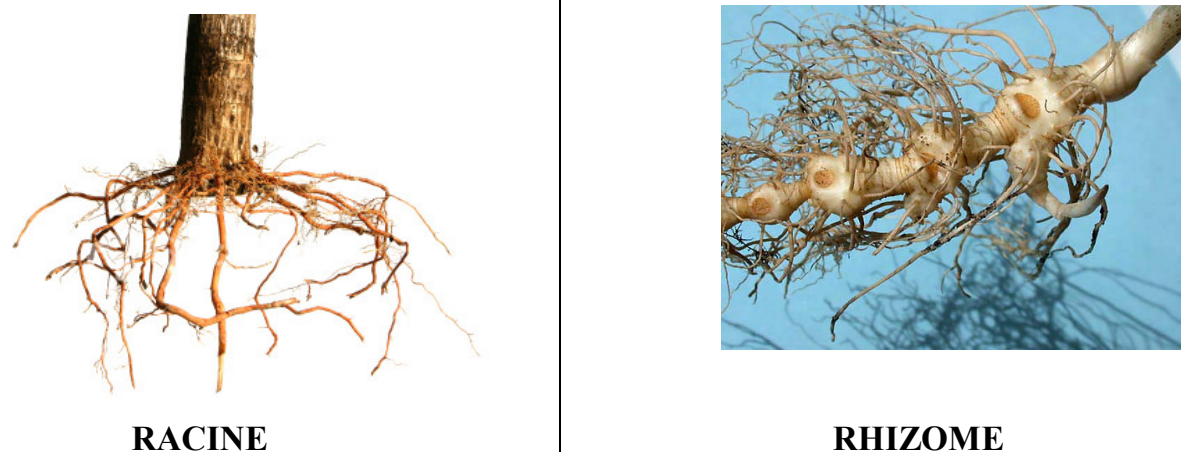

RHIZOME

\section{La mise en place d'un rhizome fictionnel dans La Bataille de Pharsale}

Le dispositif fictionnel mis en place dans La Bataille de Pharsale possède indéniablement des caractéristiques «rhizomatiques ». En effet, comme il l'expliquera lui-même en 1971 lors du colloque de Cerisy sur le Nouveau Roman, Claude Simon a choisi pour son ouvrage un schéma de construction narrative du type réticulaire (cf. fig. 2), chacun des différents «thèmes » convoqués dans le texte (cf. le commentaire qui accompagne la fig. 2) constituant un «nœud» autour duquel s'articulent plusieurs séquences narratives différentes ${ }^{1}$. De toute évidence, un tel agencement présente des similitudes frappantes avec les espaces rhizomatiques évoqués par Deleuze et Guattari, d'autant qu'il est métatextuellement décrit comme suit : «on doit se figurer l'ensemble du système comme un mobile se déformant sans cesse autour de quelques rares points fixes » $(B P, 186)$.

Or, comme tous les récits simoniens des années 1960 et 1970, La Bataille de Pharsale donne à voir un mode de composition holistique, le roman se présentant comme un vaste système architectonique où chaque détail microtextuel a été pensé et agencé en fonction de l'ensemble macrotextuel dont il fait partie intégrante : «Dans un tableau, le dessin des moindres détails participe à la composition. [...] [D]ans un texte convenablement composé, il n'y a pas de phrase qui dans ses moindres détails n'ait été écrite en fonction de l'ensemble. ") (C. Simon, lettre du 8 avril 1979, citée in Sykes 1979: 189); « [...] l'organisation de l'ensemble des éléments d'un roman ne diffère pas - sauf bien sûr par l'ampleur de l'organisation des éléments à l'intérieur d'un détail, d'une page ou d'une phrase. » (C. Simon, « La Fiction mot à mot », in Ricardou et Van Rossum-Guyon éds 1972 : 92). Dès lors, on ne sera pas étonné de voir apparaître un isomorphisme entre les structures narratives de l'œuvre et ses structures phrastiques : l'enjeu de notre étude sera de décrire le roman de 1969 comme « une totalisation en fonctionnement » (Delas et Filliolet 1973: 46), en pointant les artifices formels utilisés pour créer des agencements syntaxiques rhizomatiques qui reproduisent « en miniature » l'écheveau narratif esquissé dans la figure 2. Pour ce faire, nous montrerons que le texte simonien se caractérise par la coexistence de plusieurs ordres 
syntaxiques différents, puisque les dispositifs phrastiques rhizomatiques se retrouvent de facto imbriqués avec des dispositifs linéaires nettement plus proches des normes scripturales héritées de la tradition romanesque.

\section{FIG. 2 SCHÉMA DE COMPOSITION DE LA BATAILLE DE PHARSALE} (source : Simon $1972: 95$ )

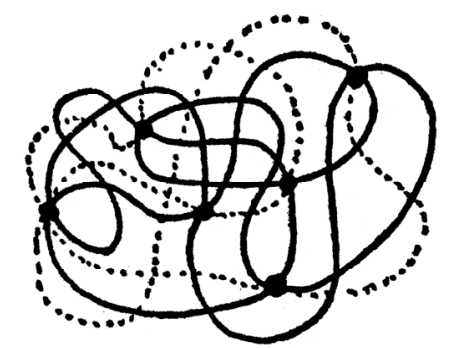

Dessin de Claude Simon

"[A]près un court prélude où les différents thèmes [narratifs] [...] sont brièvement exposés, se développe une première partie "installant" ces différents thèmes repris ensuite, approfondis avec des variations l'un après l'autre, séparément, dans la seconde partie: Batailles [Le texte porte en fait «Bataille»], Guerrier, Machine, César, Voyage, $O$ enfin [Simon oublie la sous-partie «Conversation »] [...]. Quant à la troisième et dernière partie du roman, elle rassemble une nouvelle fois les principaux thèmes et leurs dérivés, les brasse et les combine dans une polyphonie dont le tempo se fait plus rapide et haché. " (Simon 1972 : 94-95).

\section{Vers une syntaxe rhizomatique}

La tentative de mettre en place une série d'agencements scripturaux du type rhizomatique se manifeste essentiellement à travers trois phénomènes : (a) le décentrement de la phrase ; (b) l'abolition de toute forme de hiérarchie discursive ; (b) le dépassement de la linéarité syntagmatique ; (c) la reconfiguration permanente de l'ordre sémantico-syntaxique.

\subsection{Le décentrement de la phrase}

La phrase simonienne peut être qualifiée de « décentrée » dans la mesure où elle est dépourvue de points d'ancrage fixes permettant d'assigner une fonction syntaxique stable à ses différents constituants, mais aussi de reconstituer le point de vue énonciatif d'un sujet unitaire autour duquel s'articulerait la narration. Ce décentrement découle de l'application systématique de deux procédés: l'effacement des verbes conjugués, l'effacement des marqueurs d'embrayage.

\subsubsection{L'effacement des verbes conjugués}

Dans l'analyse syntaxique traditionnelle (cf. Tesnière 1959 : 102), ce sont les formes verbales conjuguées qui assument la fonction de clef de voûte de la phrase, articulant autour d'elles un certain nombre d'actants. Par sa variation en personne et en temps, le verbe conjugué rattache en principe l'action à un actant et une temporalité qui en constituent, sur des plans différents, le centre. L'effacement du « nœud» verbal contribue donc de manière décisive au « décentrement » de la phrase, puisque les actions évoquées peuvent être rattachées à des actants, des temporalités et/ou des cadres énonciatifs variables. Il en est ainsi par exemple en (2), où l'on évoque apparemment la frustration du protagoniste qui frappe de toutes ses forces à la porte de l'appartement où sa maîtresse est en train de copuler avec un autre homme (cf. BP, 57-58 et passim), mais aussi l'image d'un soldat romain dont la bouche est transpercée par une flèche ou un javelot : 
(2) entendant les battements de mon sang en afflux pressés frappant la goutte d'eau parfois l'écho assourdi d'une trompe d'auto d'un moteur je souffrais comme ailés rose vif gonflé mais pas dressé enfoncé les soldats romains étaient armés du pilum lourd javelot terminé par une courte pointe triangulaire trapue en forme de. Pilon. Bouche rose ouverte où. $(B P, 25$; nous soulignons)

La suppression quasi-totale des formes verbales conjuguées dans ce passage donne lieu à toute une série d'ambiguïtés sémantico-référentielles très visibles :

- La forme en -ANT « frappant» peut être rattachée à deux contrôleurs référentiels différents : $(\alpha)$ «les battements de mon sang» (le cœur du protagoniste pulse violemment, en raison de son émotion); $(\beta)$ «moi » (le protagoniste se rue contre la porte de la femme infidèle). Bien entendu, l'incertitude pesant sur l'actant qui est à l'origine du procès ainsi évoqué a des incidences directes sur le statut syntaxique de « frappant», qui demeure ambigu et indécidable : emploi verbal du participe ? attribut du COD «les battements de mon sang»?

- Les segments «pas dressé enfoncé », «pilon » et « bouche rose ouverte » renvoient simultanément à deux images (mentales ?) qui s'interpénètrent : celle du légionnaire atteint par un projectile, celle de la maîtresse du protagoniste qui se permet quelques familiarités avec son partenaire.

- Le segment "parfois l'écho assourdi d'une trompe d'auto" vient brouiller les repères permettant d'identifier à la fois l'énonciateur [cf. infra, point (ii)] et le contexte situationnel de la narration : en effet, le roman se déroule à une époque (la fin des années 1960) où les voitures n'ont plus des «trompes », mais des avertisseurs... On est donc en droit de se demander si en réalité la voix narrative qui résonne dans ce passage n'est pas (seulement) celle du protagoniste, mais (aussi) celle de son oncle Charles, trompé également par sa femme dans sa jeunesse ${ }^{2}$ : comme le souligne Jean Duffy (2006:1387), la fusion des expériences de l'oncle et du neveu « rend impossible toute délimitation claire et définitive entre les deux personnages ».

La syntaxe utilisée dans La Bataille de Pharsale n'est donc pas une syntaxe fondée sur le modèle de la phrase ou de la proposition avec le verbe pour élément central. Du reste, aucun des principes qui fondent la définition traditionnelle de la phrase (délimitation graphique, complétude sémantique, unité intonative, et bien sûr cohésion syntaxique) ne fonctionne pleinement chez Simon. En fait, comme on le voit bien en (2), l'écriture simonienne s'applique à faire coexister des énoncés conformes à la syntaxe traditionnelle ( «les soldats romains étaient armés du pilum » par exemple) et d'autres qui contreviennent de manière systématique, pour ne pas dire méthodique, aux principes qui fondent l'unité phrase ${ }^{3}$ : voilà pourquoi l'un de nous a été amené à proposer ailleurs (cf. Zemmour 2008) un modèle syntaxique alternatif à celui de la phrase pour rendre compte de la complexe dualité qui caractérise l'écriture simonienne. Ce modèle s'inspire des théories macrosyntaxiques notamment développées par Alain Berrendonner (cf. Berrendonner 1990, 2002, Berrendonner et Reichler-Béguelin 1989, Neveu 1998) : nous retenons en particulier la notion de clause, en remodelant toutefois les contours définitionnels de cette notion en fonction des spécificités de notre objet d'étude. Dans la définition que nous lui donnons, la clause est une unité pragma-syntaxique :

- unité pragmatique dans la mesure où elle associe un thème et un prédicat, et constitue à elle seule une unité de prédication autonome ;

- unité syntaxique dans la mesure où, un peu à la manière de la phrase - nous suivons sur ce point Berrendonner - elle constitue une « unité intégrative maximale de rection » (Berrendonner 2002: 27), ou encore un «îlot de dépendances grammaticales [...] où les éléments (morphèmes, syntagmes) sont reliés par des rapports de rection » (ibid.).

Appliqués au texte simonien, ces principes aboutissent à une segmentation de ce genre :

(3) Mains sous le kimono [/] son dos comme deux colonnes dures de part et d'autre du sillon de la colonne vertébrale [/] puis elles trouvaient cette surface plane soyeuse couverte d'un léger duvet [/] plus bas encore fesses dans mes paumes [/] un doigt entre [/] duvet [/] sa bouche disant dans la mienne oui oui oui [/] oreille qui peut voir [/] moiteur perlant [/] taisez-vous méfiez-vous des oreilles ennemies vous écoutent [/] tous les deux immobiles pétrifiés [...] $(B P, 38)$ 
On notera que notre découpage, fondé comme on l'a vu sur des principes pragmatiques et syntaxiques, est corroboré par une structure intonative récurrente, ce dont on peut faire dès lors un autre principe définitoire de la clause.

Nous avons déjà expliqué que la notion de clause englobe également la proposition. Il s'en rencontre effectivement un exemple en (3) : " puis elles trouvaient cette surface plane soyeuse couverte d'un léger duvet ». La proposition peut donc être considérée comme un type de clause particulier. Partant de cette constatation, on peut classer les clauses utilisées dans La Bataille de Pharsale en trois catégories :

- Les clauses propositionelles qui, comme celle que nous venons de citer, correspondent en fait à des propositions indépendantes.

- Les clauses participiales, dont le prédicat a pour élément central un verbe au participe (passé ou présent). Exemple : « sa bouche disant dans la mienne oui oui oui ».

- Les clauses averbales, dont le prédicat a pour élément central un élément non verbal (un adjectif, un nom, un adverbe). Exemple : « Mains sous le kimono».

Un analyse statistique opérée sur La Bataille de Pharsale permet de vérifier que Simon fait un usage équilibré des différents types de clauses : $37 \%$ pour les premières, $32 \%$ pour les secondes, $31 \%$ pour les troisièmes. Il faut signaler toutefois que, dans la troisième et dernière partie du texte, on utilise quasiexclusivement des clauses propositionnelles. À l'inverse, c'est une syntaxe averbale, fort peu académique, qui occupe les deux premiers tiers du roman de 1969. Bien entendu, ce phénomène est hautement significatif. En effet, comme le montre l'analyse de (2), l'utilisation massive de clauses participiales et averbales (63\% dans l'ensemble de La Bataille de Pharsale, avec des pointes à plus de $80 \%$ dans certains passages !) permet soit d'éliminer purement et simplement toute forme verbale susceptible de servir de «point de ralliement» sur les plans syntaxique et énonciatif, soit d'affaiblir de façon significative l'autorité du verbe, incapable à lui seul d'ordonner la phrase et de situer l'action par rapport au moment de l'énonciation et/ou à un agent ${ }^{4}$ : dans les deux cas de figure, le centre de la phrase est tout simplement absent.

\subsubsection{L'effacement des marqueurs d'embrayage}

Comme l'étude de (2) l'a laissé entr'apercevoir, la suppression des verbes conjugués permet également à Simon d'éliminer les marques discursives qui renvoient au hic et nunc énonciatif d'un Sujet narrant à la P1. Soit l'exemple suivant, où il est question d'un train qui contourne une usine :

(4) Le wagon oscillant se dandinant s'inclinant vers l'intérieur du long tournant au centre duquel dans la vallée où l'ombre commençait à s'amasser usine qui semblait pivoter lentement sur elle-même $[\ldots](B P, 161)$

De toute évidence, l'usine est vue par le narrateur à la $\mathrm{P} 1$ depuis le train au moment où celui-ci s'engage dans un virage. Toutefois, du fait que Simon s'abstient d'utiliser (comme il le fait par exemple dans $L a$ Route des Flandres) un «je pouvais voir» introduisant la chose perçue (« [je pouvais voir une] usine qui ... »), les marqueurs d'embrayage qui eussent permis au sujet narrant d'occuper le centre du récit en inscrivant son discours dans un hic et nunc énonciatif singulier font spectaculairement défaut : tous les constituants du référent fictionnel sont mis sur le même plan sans principe organisateur, le moi du narrateur semblant provisoirement néantisé.

\subsubsection{Marques métatextuelles du décentrement de la phrase}

Le décentrement énonciatif et syntaxique du texte simonien est visualisé sur le plan thématique par une mise en abyme qui a déjà été signalée par Jean Ricardou (1971 : 149-150) : il s'agit du texte (5), qui donne à voir un équivalent « miniaturisé » du récit qui l'inclut dans sa totalité.

(5) À moitié dissimulée par les herbes on distingue une plaque de tôle rouillée, en forme de triangle aux coins arrondis, creuse, comme une cuvette, percée, pas tout à fait en son centre, d'un trou rond, à peu près du diamètre d'un tuyau de poêle, par où s'élance une épaisse touffe d'herbe. $(B P, 231)$ 


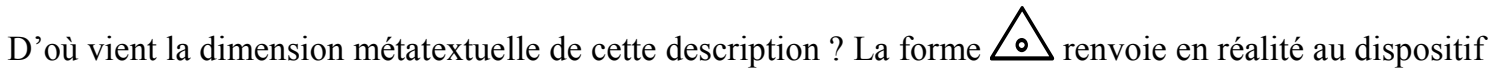
fictionnel mis en place dans La Bataille de Pharsale. Comme l'a brillamment montré Stuart Sykes (1979 : 134 ; cf. fig. 3), ce dispositif se présente comme un triangle au centre duquel se trouve la lettre O, utilisée pour désigner en même temps les trois principaux personnages : le protagoniste, sa maitresse infidèle, son oncle Charles. Or, cette lettre figure clairement un espace vide, un pure absence ${ }^{5}$ l'univers narratif mis en place par Simon est proprement «acentré », dans la mesure où la forme $\triangle 0$ renvoie à la fois

- à la disparition du Dieu chrétien (symbolisé, comme on le rappelle fort opportunément dès la p. 10, par un triangle avec un œil au milieu) ;

- à la destruction de l'identité des trois personnages qui occupent le centre de la scène narrative, ces derniers « fusionnant » partiellement entre eux tout comme les composantes de la Sainte Trinité ;

- à la néantisation corrélative du moi narrant, qui apparaît comme une forme vide susceptible de désigner à la fois le protagoniste et son oncle (« et moi au centre $», B P, 117)$.

\section{FIG. 3 SCHÉMA DE COMPOSITION DE LA BATAILLE DE PHARSALE (source : Sykes $1979: 134)$}

LA BATAILLE DE PHARSALE Recherche et souvenir ensemble

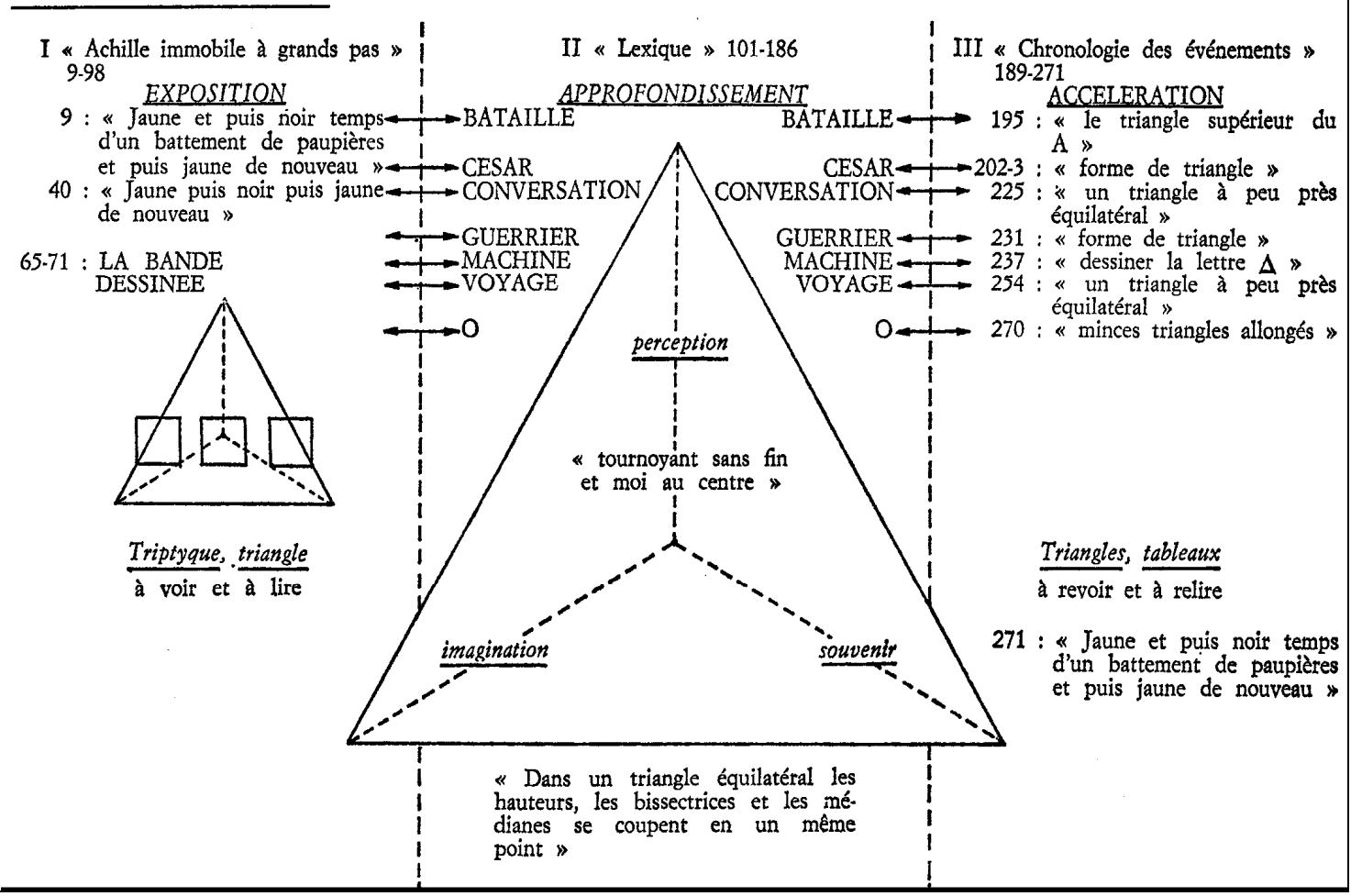




\subsection{L'abolition de la hiérarchie discursive}

Pour conférer à son écriture une dimension rhizomatique, Simon utilise une série de procédés qui permettent d'abolir ou à tout le moins de remettre en question les schémas syntaxiques fortement hiérarchisés hérités des grammaires normatives des XVIII ${ }^{\mathrm{e}}$ et $\mathrm{XIX}^{\text {ème }}$ siècles et érigés en norme stylistique par les romanciers « classiques ». Quels sont ces procédés ? On retiendra ici l'autonomisation d'éléments syntaxiquement régis, l'emploi « déviant» des connecteurs, qui permettent de coordonner entre eux des éléments hétérogènes, et le travail des subordonnants (conjonctions de subordination, prépositions, pronoms relatifs), qui tendent à fonctionner comme des connecteurs.

\subsubsection{L'autonomisation d'éléments syntaxiquement régis}

Des éléments a priori dépendants sur le plan syntaxique accèdent dans certains passages à une forme d'autonomie (cf. Zemmour 2008 : 126-129, 318 et passim). Il en est ainsi par exemple dans l'extrait suivant, où le narrateur, accompagné de son ami Nikos, se met à chercher le site de la bataille de Pharsale et tente de se rappeler si, chemin faisant, il n'aurait pas croisé un terrain de football :

(6) À la réflexion il y en avait un une partie se déroulait puisqu'il m'a dit que j'allais nous flanquer dans le fossé et alors j'ai arrêté l'auto et pendant un bon moment nous les avons regardés courir et se poursuivre je peux me rappeler le bruit mat quand ils tapaient dans le ballon $[\ldots](B P, 97)$

La construction syntaxique de cette longue phrase pose clairement problème. En effet, si l'on restitue le processus psychomémoriel apparemment évoqué par le moi narrant, on devrait avoir : « À la réflexion il y en avait un une partie se déroulait puisque [je me souviens qu']il m'a dit que j'allais nous flanquer dans le fossé et [qu']alors j'ai arrêté l'auto et [que] pendant un bon moment nous les avons regardés courir et se poursuivre je peux me rappeler le bruit mat quand ils tapaient dans le ballon ». Or, du fait de l'ellipse de l'élément recteur «je me souviens que », on passe insensiblement du monologue remémoratif ${ }^{6}$ ("À la réflexion il y en avait un une partie se déroulait ») au récit à la $\mathrm{P} 1$ pur et simple, les segments phrastiques «alors j'ai arrêté l'auto » et « pendant un bon moment nous les avons regardés courir et se poursuivre » tendant de facto à s'affranchir de toute tutelle sur le plan énonciatif et syntaxique : l'effacement de la proposition rectrice travaille à l'abolition de la hiérarchisation référentielle et discursive qui était devenue la règle dans le roman «classique », dont les normes scripturales sont ici délibérément transgressées.

\subsubsection{L’emploi « déviant » des connecteurs syntaxiques}

Cette abolition passe aussi par un emploi sui generis des connecteurs syntaxiques, qui permettent de coordonner entre eux des constituants phrastiques de statut syntaxique, sémantique et/ou énonciatif différent (cf. Zemmour 2008: 76). Ainsi justement en (6) la conjonction «et» est utilisée «contre nature ", puisqu'elle relie entre eux deux constituants phrastiques qui ne se trouvent pas sur le même plan : une subordonnée stricto sensu («puisqu'il m'a dit que j'allais nous flanquer dans le fossé »), une subordonnée fonctionnant en réalité comme une proposition non dépendante ( «alors j'ai arrêté l'auto »). Bien entendu, la mise en place de connexions de ce genre, qui consacre sur le plan stylistique le triomphe de l'« unité du multiple » dans le récit simonien (cf. Deleuze 1968 : 59, Yocaris 2002: 134 et passim), est une composante sine qua non de tout agencement rhizomatique digne de ce nom : en effet, comme le soulignent Deleuze et Guattari, le rhizome, du fait qu'il a vocation à relier entre eux des éléments fondamentalement hétérogènes, « a pour tissu la conjonction et... et... et... » (Deleuze et Guattari 1980 : 36).

\subsubsection{La transformation des subordonnants en connecteurs d'énoncés autonomes}

Soit l'exemple suivant : 
(7) montagne son énorme dos courbé montagne de viande courbée sur elle avec cette peau trop blanche blafarde et cette tignasse rousse que je n'avais jamais pu dissocier de la casquette de marinier hollandais me demandant si une seule fois il m'était arrivé de le voir sans elle, comme si elle était collée à sa tête, comme si elle faisait partie intégrante de son personnage au même titre que la barbe et les favoris [...] $(B P, 75$; nous soulignons)

Dans cet exemple, les unités phrastiques introduites par la locution conjonctive « comme si » (« comme si elle était collée à sa tête », « comme si elle faisait partie intégrante de son personnage ») tendent de facto à s'autonomiser, alors qu'il s'agit en principe de constituants syntaxiquement dépendants : «comme si » ne fonctionne plus en l'occurrence comme un marqueur de subordination introduisant une comparative hypothétique, mais comme un connecteur reliant entre elles des «clauses » énonciatives situées plus ou moins sur le même plan ${ }^{7}$.

\subsubsection{Marques métatextuelles de la dé-hiérarchisation syntaxique}

La dé-hiérarchisation de l'espace narratif et syntaxique dans La Bataille de Pharsale peut être considérée comme la manifestation stylistique la plus visible d'une forme d'anarchisme conceptualisé ${ }^{8}$ (cf. Yocaris 2002 : 316-317), Simon tâchant de construire un univers fictionnel typiquement postmoderne où l'on met « tout sur le même plan » (L'Herbe, p. 123), où il n'y a plus aucun point de vue privilégié sur le monde, toute autorité, toute préséance politique, religieuse, esthétique, culturelle ou morale ayant été abolies. Une telle démarche est notamment abymée par une série de remarques portant sur les techniques stylistiques des peintres Allemands de la Renaissance, que Simon affectionnait tout particulièrement (cf. p. ex. Simon 1977 : 32). Voici la teneur de ces remarques, extraites en fait de l'Histoire de l'art d'Élie Faure :

(8) O. lit dans une Histoire de l'Art le chapitre sur les peintres allemands de la Renaissance : "Jamais ils ne vont par le plus droit chemin au seul essentiel et au plus logique. Le détail masque toujours l'ensemble, leur univers n'est pas continu, mais fait de fragments juxtaposés. On les voit, dans leurs tableaux, donner la même importance à une hallebarde qu'à un visage humain, à une pierre inerte qu'à un corps en mouvement, dessiner un paysage comme une carte de géographie, apporter, dans la décoration d'un édifice, autant de soins à une horloge à marionnettes qu'à une statue de l'Espérance ou de la Foi, traiter cette statue avec les mêmes procédés que cette horloge, et quand ..." O. sort de sa poche un stylo-mine et écrit dans la marge : Incurable bêtise française. ( $B P, 238$; cf. $B P, 174$, Faure $1976: 345-346)$.

\subsection{Le dépassement de la linéarité syntagmatique}

La dimension dé-hiérarchisée de la syntaxe simonienne favorise le développement d'interconnexions complexes et de «systèmes de renvois » multidirectionnels, au détriment des agencements syntagmatiques plus ou moins linéaires hérités de la tradition grammaticale (cf. Yocaris 2006b : 223224). Bien entendu, ces agencements ne sont pas entièrement éliminés dans La Bataille de Pharsale, loin de là, mais ils coexistent avec des dispositifs phrastiques dont les composantes ne se suivent pas (ou pas seulement) de façon univoque mais entretiennent mutuellement des rapports d'imbrication, de chevauchement et/ou de superposition. Parmi les phénomènes concernés par cette «nouvelle donne » scripturale, on retiendra surtout l'émergence de connexions syntaxiques adventives, l'utilisation d'hyperbates, les procédés relevant d'une "syntagmatisation du paradigmatique » et enfin, last but not least, les effets de « segmentation floue ».

\subsubsection{Le développement de connexions syntaxiques adventives}

C'est là une caractéristique propre aux rhizomes végétaux : une racine adventive est une racine qui pousse à un endroit imprévisible de la tige, contribuant ainsi à la multiplication et au bouturage des plantes (cf. fig. 4). Les racines de ce type apparaissent donc là où elles n'auraient pas dû apparaître, où rien 
n'indiquait a priori qu'elles eussent pu pousser. Or, le même phénomène apparaît, mutatis mutandis, au niveau de la construction phrastique du texte simonien. Soit l'exemple suivant :

(9) la couche des ténèbres s'amincit achève peu à peu de se diluer transparentes s'égouttant lentement [...] (BP, 162 ; nous soulignons)

C'est l'adjectif « transparentes » qui nous intéresse ici. En effet, «transparentes » caractérise visiblement " ténèbres », avec lequel il s'accorde en genre et en nombre. Or, ce choix syntaxique est tout à fait inattendu, dans la mesure où le caractérisant «transparentes» apparaît après un couple de groupes verbaux ( «s'amincit achève peu à peu de se diluer ») ayant pour sujet le SN «la couche », et non point le $\mathrm{SP}$ «des ténèbres » (qui dépend de ce $\mathrm{SN}$ ). On est donc confrontés en l'occurrence à une construction syntaxique particulièrement transgressive, puisque le segment phrastique «transparentes s'égouttant lentement» prend appui sur un constituant syntaxiquement second («ténèbres») entrant dans la formation d'un complément du nom qui n'était pas voué en principe à faire l'objet de nouvelles expansions : il est pourtant le point de départ d'une nouvelle «ramification» textuelle.

\section{FIG. 4}

RACINES ADVENTIVES

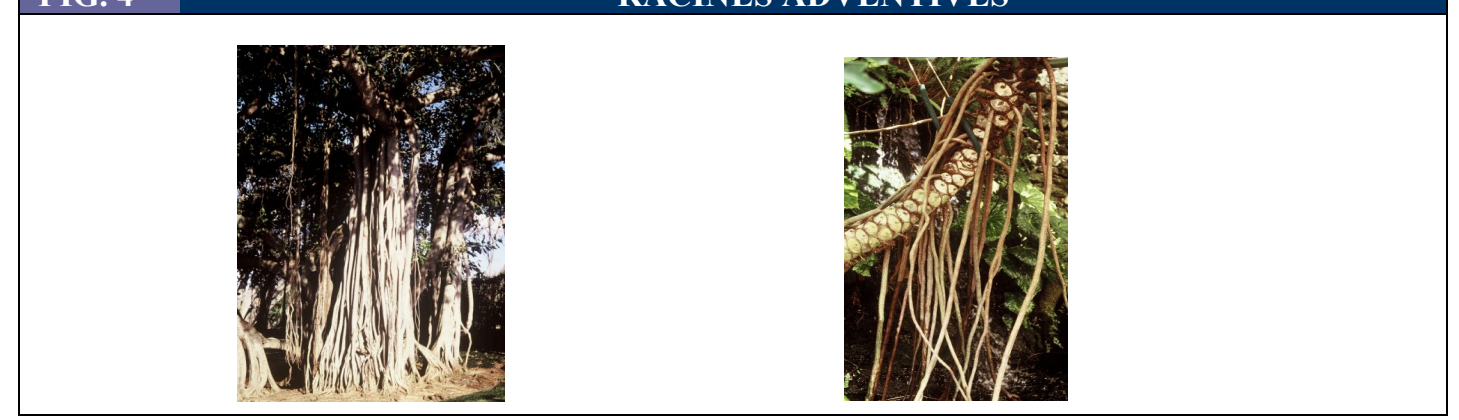

\subsubsection{Le travail de l'hyperbate}

Dans son acception la plus courante, l'hyperbate est un prolongement inattendu de la phrase après une clôture syntaxique qui en marque normalement la fin (cf. Mazaleyrat et Molinié 1989 : 170). Ainsi, pour reprendre un exemple bien connu, dans « Albe le veut, et Rome », l'effet propre à l'hyperbate vient de ce que l'élément ajouté fait partie du syntagme assumant la fonction sujet, alors que la présence du verbe indiquait que l'expression du sujet était close. L'hyperbate suppose donc dans ses acceptions habituelles la notion de phrase achevée, puisqu'elle vient par définition démentir cet achèvement. Voyons ce qu'il en est chez Simon :

(10) Bataille quel peintre allemand devant des feuillages vert noir cartonneux au pied de rochers escarpés ou plutôt séance d'abattage bûcherons dans des armures de métal blanc guillochées avec ces coudes comme ont les tuyaux de descente ces coquilles Saint-Jacques bombées entre les jambes massacrant comme on déboise maniant à deux mains leurs lourdes rapières comme des ouvriers [...] $(B P, 176$; nous soulignons ; italiques du texte)

On observe dans ce passage deux hyperbates. Tout d'abord, la clause averbale «ou plutôt séance d'abattage » vient après coup se raccorder à «bataille » («Bataille [ou plutôt séance d'abattage] »), pardelà la clôture syntaxique matérialisée par les clauses «devant des feuillages vert noir cartonneux » et « au pied de rochers escarpés »: le texte transgresse de la sorte les catégories ontologiques du dedans et du dehors, ce qui est une caractéristique typiquement rhizomatique (cf. Deleuze et Guattari 1980:66). Ensuite, la forme en -ANT «massacrant» se rattache en fait à «bûcherons », quelques lignes plus haut, alors que le texte semblait en avoir fini avec les chevaliers flatteusement désignés par ce terme pour passer à la description de leurs armures... Des hyperbates de ce genre confèrent au récit simonien une 


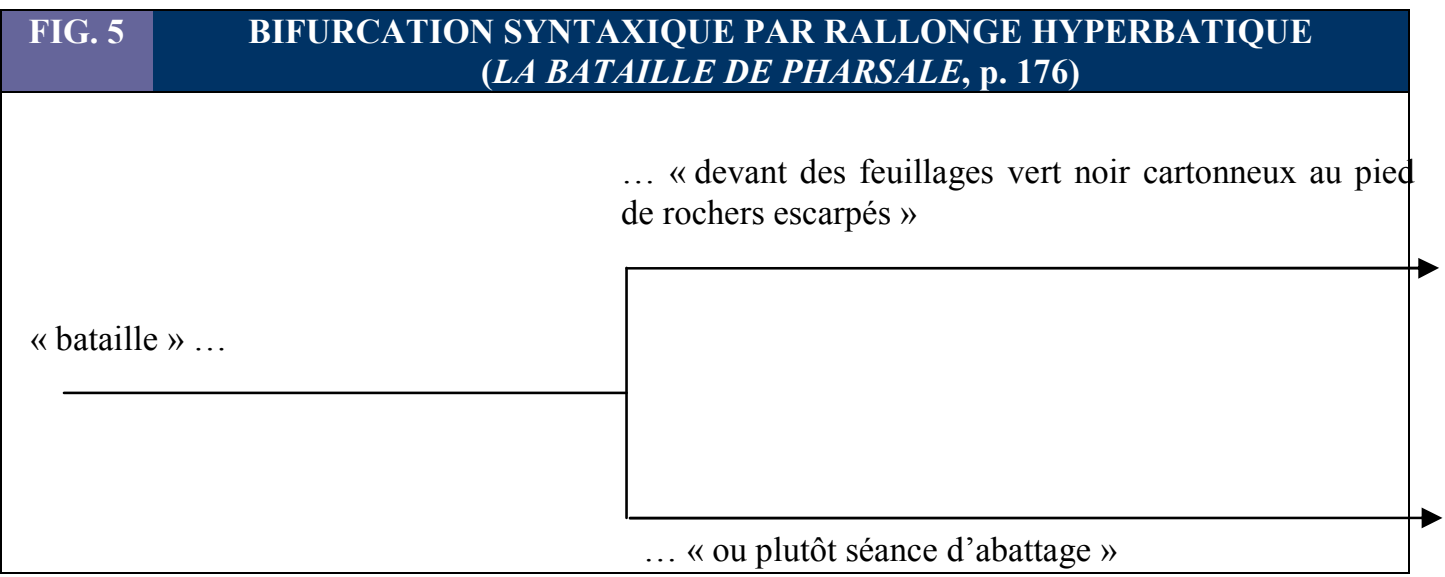

\subsubsection{La syntagmatisation du paradigmatique}

Dans des romans comme La Route des Flandres ou La Bataille de Pharsale, Simon fait se succéder des mots ou expressions qui, étant de sens voisin, entretiennent entre eux une relation de substitution possible et à ce titre appartiennent au même paradigme (cf. Zemmour 2008 : 120). Il en est ainsi par exemple dans l'incipit du roman de 1969 [cf. texte (1)], où l'on évoque un oiseau dont l'envol rappelle au moi narrant un tableau qui présente des similitudes frappantes avec La Bataille de Zara de Tintoret (1584-1587): «peut-être pas entendu perçu rien qu'imaginé oiseau flèche fustigeant fouettant déjà disparue l'empennage vibrant les traits mortels s'entrecroisant dessinant une voûte chuintante comme dans ce tableau vu où ? combat naval entre Vénitiens et Génois sur une mer bleu-noir crêtelée épineuse ». Quelle analyse proposer des couples de lexèmes et/ou de syntagmes de sens voisins que nous avons soulignés ici ? On pourrait simplement considérer que l'on se trouve dans une logique psychologique de la rectification, le narrateur étant à la recherche du mot juste. Une telle analyse est assurément recevable, mais sa portée reste locale. En effet, si dans La Bataille de Pharsale le narrateur ou le héros semble bien quelquefois chercher l'expression adéquate pour désigner tel ou tel constituant du référent fictionnel (cf. par exemple $B P, 23,40$ et passim), dans la plupart des cas les mots et expressions appartenant à un même paradigme forment un bloc indivisible: c'est ce qui se passe en (1) avec le couple «crêtelée épineuse », qui permet de faire ressortir simultanément deux sèmes (/aspect houleux/, /forme pointue/) dont l'un est absent du sémème de chaque mot pris isolément. Le texte travaille dans ce cas de figure non point à la recherche du mot juste, mais à l'élaboration de la signification adéquate par le recours à tout un complexe lexical dont les composantes entrent mutuellement en résonance : de la sorte, le lecteur n'a pas accès à un mot et/ou une signification unitaire(s), mais à l'appréhension instantanée de toute une multiplicité scripturale visant à reproduire la mouvante complexité du réel.

\subsubsection{La segmentation floue}

Nous empruntons cette notion à Claire Blanche-Benveniste et Colette Jeanjean (1987: 141) : celles-ci montrent que, dans un énoncé comme «ça y est il coulait le mois d'après il était au chômage », le segment « le mois d'après » peut être rattaché aussi bien à son cotexte gauche (« ça y est il coulait le mois d'après ») qu'à son cotexte droit (« le mois d'après il était au chômage »). Alain Berrendonner envisage 
lui aussi le cas de fragments discursifs qui se prêtent à une double analyse de ce genre, qu'il nomme «métanalyse » (Berrendonner 2002 : 33-34). Chez Simon, une telle pratique est à la fois fréquente et consciente d'elle-même (cf. Yocaris 2006b: 230-232, Zemmour 2008: 82 et passim). Pour s'en convaincre, il suffit de se pencher sur le texte (11):

(11) Dans L'Énéide ou quoi ? Quelque chose d'obscur marron un ciel cuivré avec des fumées rougeoyantes dans le fond des éclats métalliques les reflets orange et noirs des incendies sur les flancs des cuirasses les yeux noyés révulsés des femmes tordant leurs bras et celle fuyant qui se retourne pour regarder derrière elle le visage éclairé par les flammes et celui qui portait le vieillard barbu sur ses épaules et la mer amère noire fouettant le goût du sel sur les lèvres les récifs noirs aux noms de monstres cernés d'écume et la déesse apparaissant dans les nuées coiffée d'un casque de bronze [...]. $(B P, 17$; nous soulignons)

Dans cet extrait, où l'on évoque apparemment une scène de l'Énéide (Énée porte Anchise sur ses épaules pour l'éloigner de la ville de Troie en proie aux flammes), on repère d'emblée une anomalie syntaxique (parmi d'autres, qui seront traitées plus loin) : le groupe prépositionnel «dans le fond» appartient à la fois à l'unité discursive qui précède ( « des fumées rougeoyantes dans le fond ») et à celle qui suit (« dans le fond des éclats métalliques »). La logique de la linéarité syntaxique est ici compromise par un phénomène de chevauchement : de façon symptomatique, un phénomène similaire est pointé par Deleuze et Guattari lorsqu'ils expliquent qu'un espace rhizomatique comporte nécessairement des «zones limitrophes et transitionnelles, d'indiscernabilité » (Deleuze et Guattari 1980 : 128).

\subsubsection{Marques métatextuelles du dépassement de la linéarité}

La dimension non linéaire de la syntaxe simonienne trouve son expression thématique la plus marquante dans une mise en abyme qui constitue à n'en point douter un vrai topos dans les œuvres des Nouveaux Romanciers, toutes tendances confondues: il s'agit de la description des rails du chemin de fer, qui s'entrecroisent sans cesse.

(12) les rails se divisant bruyamment s'écartant bifurquant se rapprochant divergeant de nouveau se dédoublant encore se multipliant s'étalant sur une grande surface $(B P$, 164)

\subsection{La reconfiguration permanente de l'ordre sémantico-syntaxique}

Reste à évoquer le trait distinctif le plus important de la syntaxe rhizomatique, à savoir l'aspect protéiforme des structures phrastiques : en effet, il est clair que le travail scriptural entrepris dans $L a$ Bataille de Pharsale vise avant tout à mettre en évidence « le développement continu d'une forme qui ne cesse pas de se dissoudre ou de se transformer » (Deleuze et Guattari 1980:120). Le texte simonien devient un perpetuum mobile, puisque la fonction des éléments qu'il met en relation, leur nature et leur position se modifient en même temps que les rapports qui les relient entre eux. Le lecteur se trouve dès lors confronté à une série d'agencements syntaxiques "à géométrie variable », qui doivent être saisis dans un processus de reconfiguration permanente. La labilité de l'ordre sémantico-syntaxique ainsi établi se manifeste essentiellement à travers trois phénomènes: la mise en place d'une hiérarchie phrastique réversible, les effets d'anamorphose syntaxique et l'instauration d'un système de règles variables.

\subsubsection{La mise en place d'une hiérarchie phrastique réversible}

Un même segment syntaxique peut faire l'objet d'analyses différentes, mais simultanément possibles. Il peut ainsi être considéré à la fois comme élément de prédication principale et secondaire. Prenons par exemple la citation suivante :

(13) puis elle [l'usine] disparut le train roulant toujours la vallée cessant bientôt les montagnes cessant le soleil entrant de nouveau dans le compartiment les rayons plus obliques maintenant $[\ldots](B P, 162)$ 
Dans ce segment phrastique, le syntagme «le train roulant toujours» peut s'analyser comme une proposition participiale dépendant de la principale «elle disparut», avec une valeur causale et/ou temporelle. Mais la suite du discours oblige à envisager également une autre analyse, sans pour autant invalider la première : l'accumulation de constructions similaires ( "la vallée cessant bientôt», «les montagnes cessant », « le soleil entrant de nouveau dans le compartiment ») invite en effet le lecteur à considérer qu'il a affaire à des unités de prédication autonomes, des «clauses participiales » qui n'ont certes pas la forme de propositions indépendantes (puisqu'elles ne sont pas construites à partir d'un verbe conjugué), mais qui paraissent fonctionner de la même manière. Dès lors, par attraction, le syntagme «le train roulant toujours » apparaît lui aussi après coup comme une clause participiale sémantiquement et syntaxiquement autonome, cette lecture du texte venant se superposer à la précédente : en termes d'effet produit, on se trouve ici confrontés à un phénomène d'indécidabilité syntaxique, comme si premier plan et arrière-plan, fait saillant et fait secondaire étaient totalement réversibles. On voudra bien noter que la « réversibilité » des structures phrastiques du récit simonien se trouve dûment thématisée par une série de références métatextuelles à des « objets complexes » en forme de «cubes de Necker» (cf. BP, 156/160161, 220 ; Yocaris $2002: 331-333$; fig. 6) : l'évocation de ces objets inscrit, au cœur même de la matière fictionnelle, « le principe de la réversibilité des perspectives » (Duffy $2006: 1401$ ).

FIG. 6

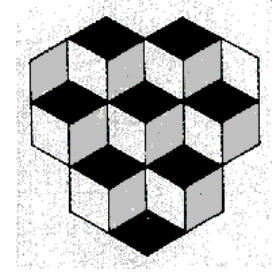

\subsubsection{Les effets d'anamorphose syntaxique}

Le « remodelage » constant des contours de la phrase simonienne aboutit dans certains cas de figure à de véritables anamorphoses sur le plan syntaxique : on voit ainsi émerger dans La Bataille de Pharsale des agencements discursifs dont les composantes n'ont pas des propriétés stables sur le plan énonciatif, syntaxique et/ou narratif, mais "se définissent seulement par un état à tel moment [de la lecture] » (Deleuze et Guattari 1980: 26; italiques des auteurs). Reprenons le texte (11): dans cet extrait, on s'intéressera cette fois-ci aux pronoms démonstratifs soulignés ( « et celle », « et celui »). Le premier (« et celle fuyant ») renvoie anaphoriquement à l'une des femmes qui prend la fuite. En revanche, le deuxième (« et celui qui portait le vieillard barbu ») ne se réfère directement à aucun élément mentionné en amont ou en aval : il s'agit donc d'un déictique renvoyant par exophore mémorielle à un élément connu du narrateur et du lecteur, à savoir l'image d'Énée portant son père. Le fait que les deux démonstratifs soient coordonnés par le biais d'une polysyndète alors que leur statut énonciatif n'est a priori pas le même constitue certes une transgression qui renvoie directement à l' «instauration d'une logique du ET» (Deleuze et Guattari $1980: 37$ ) propre aux espaces rhizomatiques. Mais ce n'est pas tout! Le segment " et celle fuyant qui se retourne pour regarder derrière elle » semble bien dans ce contexte convoquer aussi un extrait de l'Ancien Testament, à savoir la description de la femme de Loth : celle-ci fut changée en colonne de sel au moment précis où elle se retournait pour contempler la destruction de Sodome et de Gomorrhe, réduites en cendres par une pluie de feu (Genèse, 19, 15-28). La convocation implicite de l'hypotexte biblique semble motivée par trois éléments: (a) l'incertitude qui pèse de toute façon sur l'origine de la scène décrite («Dans l'Énéide ou quoi ? »); (b) la prégnance dans La Bataille de Pharsale de l'hypotexte proustien, qui fait une place de choix à l'image de Sodome et de Gomorrhe; (c) l'homonymie implicite « celle » / « sel» ... Or, aucune référence à l'Ancien Testament n'est proposée dans le texte (11). Dès lors, il devient clair que le statut référentiel et syntaxique du démonstratif « celle » a évolué au fil des mots : ce qui est au départ un anaphorique «pur» renvoyant par association 
synecdochique à «femmes»(dans le cotexte gauche) se transforme bien vite en une forme mixte, à la fois anaphorique et déictique, puisque «celle » convoque également, par exophore mémorielle, l'histoire de la femme de Loth (censée être présente dans l'esprit du narrateur en même temps que la scène de la fuite d'Énée); cette première transformation est suivie d'une deuxième, puisque le «déicticoanaphorique » « celle » redevient « simple » anaphorique à hauteur du segment « et celui qui portait le vieillard barbu », qui oriente de nouveau le récit vers l'hypotexte virgilien. Le lecteur se trouve donc confronté en l'occurrence à un enchaînement d'anamorphoses syntaxiques dont chacune modifie du tout au tout la structure énonciative et - par extension - la portée référentielle du texte simonien : au fur et à mesure que progresse la lecture, la forme verbale «celle» «change d'état» en permanence, reconfigurant du coup l'ensemble de l'agencement discursif qui l'inclut, et qui s'avère composé d'éléments à la fois homogènes et hétérogènes.

\subsubsection{La variabilité des règles}

On retrouve cette tension entre homogénéité et hétérogénéité au niveau métasyntaxique, puisque les normes scripturales présidant à la constitution du discours narratif dans La Bataille de Pharsale sont elles-mêmes instables. Il va de soi que cette variabilité de la règle, au sens strictement linguistique du terme ${ }^{9}$ est une caractéristique rhizomatique par excellence, Deleuze et Guattari remarquant que la constitution d'un rhizome peut être décrite comme « un jeu où chaque coup porterait sur la règle » (1980: 126). Comment se manifeste concrètement cette variabilité dans La Bataille de Pharsale ? Le lecteur découvre assez vite que l'emploi des signes de ponctuation, mais aussi la disposition graphique du texte (découpage en paragraphes, alternance romains/italiques) n'obéissent pas à un seul système de règles, fussent-elles propres au récit simonien, mais à plusieurs systèmes concurrents, qui alternent de façon en apparence aléatoire (cf. Bikialo 2000). Ainsi par exemple, pour délimiter les différentes unités phrastiques, Simon utilise et croise toutes les variantes possibles: absence/ présence de majuscule, absence / présence de ponctuation. Il en va de même pour les fragments citationnels insérés dans le texte : a priori, ces fragments sont différenciés de l'hypertexte simonien du fait qu'ils sont cités en italiques. Il se crée ainsi peu à peu dans la première partie de La Bataille de Pharsale une "norme macrocontextuelle ${ }^{10}{ }^{1}$ que le lecteur avalise sans même s'en apercevoir : à chaque fois qu'il voit un passage en italiques, il aura fatalement tendance à le considérer comme une citation insérée dans le « récit principal » en romains. Or, comme on pouvait s'y attendre, Simon ne se prive pas de transgresser cette norme : dans la deuxième partie du roman, la distribution romains-italiques se trouve par moments inversée. Ainsi, dans la sous-partie «Voyage», quelques fragments de la Recherche sont graphiés en romains, comme s'ils faisaient partie intégrante du «récit» principal (v. $B P, 155,158,181)$; inversement, dans « Bataille », une part non négligeable de ce « récit » est présentée en italiques, comme s'il s'agissait en l'occurrence d'insertions intertextuelles (v. BP, 102, 104, 106, 107-108, 109, 109-110, 110, 110-112 etc.). De la sorte, la distinction entre le dedans et le dehors se trouve une fois de plus abolie, le récit simonien illustrant à tous les niveaux de sa construction le principe déjà mentionné de la « réversibilité des perspectives ».

\subsubsection{Marques métatextuelles de l'instabilité syntaxique du texte}

La reconfiguration permanente de la phrase simonienne, dont «chaque élément ne cesse pas de varier et de modifier sa distance par rapport aux autres » (Deleuze et Guattari 1980:43) se trouve thématisée par une mise en abyme des plus significatives et des plus élaborées, à savoir... la description d'une bouteille de soda :

(14) refusant le gobelet de carton buvant à même la bouteille d'un brun orangé [...] le vide dans la partie supérieure de la bouteille au-dessus du liquide brun rempli par un cloisonnement de grosses bulles aux fines membranes comme des bulles de savon Le soleil brillait sur les parois translucides des bulles De temps en temps l'une d'elles éclatait l'ensemble des cellules transparentes s'organisant aussitôt dans une autre structure mailles d'un filet en tous sens $(B P, 167-168)$ 
La description des bulles contenues dans la bouteille est de toute évidence une mise en abyme du récit simonien, dans la mesure où elle permet justement de visualiser l'instabilité et la complexité proprement rhizomatiques de ses structures narratives et phrastiques. On remarque en effet que les bulles forment un « ensemble [de] cellules transparentes » dont les éléments constitutifs, inextricablement imbriqués, font partie intégrante d'un réseau de connexions multidirectionnelles et se modifient constamment au fur et à mesure qu'évolue la configuration globale de la structure qui les contient.

\section{Rhizome et poétique du roman postmoderne}

La mise en place d'un agencement rhizomatique dans La Bataille de Pharsale n'est visiblement pas un phénomène isolé qui aurait émergé ex nihilo. En effet, force est de constater que les choix scripturaux effectués sans doute de façon intuitive par Simon correspondent parfaitement aux invariants narratifs et stylistiques qui se dégagent quand on étudie les œuvres de fiction postmodernes en général (cf. notamment Hassan 1982, 1987, McHale 1987, Hutcheon 1988, Fokkema 1997, Connor 2004). D’où vient cette similitude? Du fait que le récit simonien est structuré comme un rhizome et se donne à voir comme tel via un réseau de mises en abyme parfaitement cohérentes, il dénote et exemplifie en même temps ${ }^{11}$ une série de prédicats ( multiplicité », « incertitude », « instabilité », 《 anamorphose », « aspect labyrinthique », « mouvement permanent», «éclatement», « interconnexion», « ramification», « hétérogénéité », « hybridation », « décentrement » etc.) qui constituent autant de lieux communs de la critique et de la littérature postmodernes, toutes tendances confondues. La poétique du roman postmoderne repose en effet grosso modo sur les traits distinctifs suivants :

- Une absence d'unité, puisqu'on tient à rendre compte de la disparition de tout principe philosophique, esthétique, épistémologique, anthropologique, moral ou métaphysique susceptible d'ordonner le « réel » dans sa totalité et de lui donner un sens univoque.

- Une absence de toute forme de hiérarchisation des données textuelles.

- Une esthétique de l'hybridation, de l'empiètement (impingement), privilégiant la mise en rapport d'éléments hétérogènes strictement placés sur le même plan (cf. Connor 2004 : 77).

- Une vision du monde axée sur l'indétermination ontologique

- Un agencement non linéaire des constituants narratifs et/ou verbaux, les textes postmodernes oscillant entre la non connectivité (absent connectivity), la sous-connectivité (under-connectivity) et la surconnectivité (over-connectivity) sur le plan structural (cf. Fokkema 1997 : 39, Connor 2004 : 78 ).

- Une fascination pour le mouvement, le devenir sous toutes ses formes.

L'étude de l'organisation discursive dans La Bataille de Pharsale montre donc que ce roman appartient de droit à la mouvance postmoderne, de même que l'ensemble des Nouveaux Romans simoniens des années 1960 et 1970 (à l'exception peut-être du Palace). Pour synthétiser tout ce qui précède, on dira que les caractéristiques «rhizomatiques» de l'écriture simonienne peuvent être considérées comme des formes de « connectivité narrative instable» (unstable narrative connectivity : cf. Fokkema $1997: 39$ ), et à ce titre rattachées à l'idée typiquement postmoderne d'un monde irrémédiablement fragmenté où seule prévaut l'unité paradoxale du multiple : de même que l'univers qu'il est censé décrire, un roman comme La Bataille de Pharsale n'est pas réductible dans sa totalité à un ordre totalisant et immuable, mais se trouve structuré par une série d'agencements hétérogènes de portée strictement «locale » qui se superposent en partie, se complètent et/ou s'excluent mutuellement en même temps ${ }^{12}$.

On en arrive ainsi à ce qui fait la quintessence de l'œuvre sur le plan référentiel : bien plus qu'à une allégorie autoréflexive, une sorte de «monologue remémoratif » ou un ouvrage autobiographique relatant «à mots couverts» certains épisodes de la vie intime de son auteur comme l'ont signalé certains critiques, nous avons affaire en l'occurrence à un «objet» verbal complexe qui fonctionne comme un modèle cognitif (cognitive map). Du fait de sa dimension rhizomatique, le dispositif textuel mis en place dans La Bataille de Pharsale ne peut plus être considéré comme un simple « reflet» du «monde extérieur» (un «calque» dans la terminologie deleuzienne ${ }^{13}$ ), puisqu'il forme lui-même un monde en miniature, possédant ses propres lois de fonctionnement (en termes deleuziens, on dira qu'il devient une «carte $\left.{ }^{14}\right)$. Claude Simon rejoint ainsi la démarche d'un auteur typiquement postmoderne comme Italo Calvino. Ce dernier appelle effectivement de ses vœux « une littérature du défi au labyrinthe » (Calvino 
2003 : 115), visant à «exprimer la multiplicité cognitive du monde dans lequel nous vivons 》 (ibid. : 109) : l'enjeu fondamental de l'écriture romanesque dans une telle optique est la création de rhizomes textuels de toutes sortes (cf. ibid.: 114-115), dont l'exploration est censée permettre au lecteur d'«affronter la complexité du réel, en refusant les visions simplistes qui ne font que confirmer nos habitudes de représentation du monde » (ibid.: 115). Pour Simon comme pour Calvino, la mise en place d'un rhizome textuel relève in fine d'un pari gnoséologique : il s'agit d' ' éduquer » en quelque sorte le lecteur en faisant en sorte que ce dernier, systématiquement confronté à des «objets» textuels intrinsèquement ambigus, indéterminés et complexes, apprenne peu à peu à les «manier » et finisse ainsi par en reconnaître les "propriétés » fondamentales. Il va de soi que l'on retrouve la même conception de l'écriture chez d'autres auteurs plus ou moins proches de la mouvance postmoderne comme Eco, Borges, Cortázar, Butor ou Robbe-Grillet. ${ }^{15}$

\section{Conclusions (méta)théoriques}

Notre approche permet avant tout de rendre compte de la dimension holistique de La Bataille de Pharsale: en effet, les constituants du roman de 1969 ne peuvent être considérés isolément, mais uniquement en fonction de leur appartenance à un ensemble de structures rhizomatiques dont ils font partie intégrante. Chacune de ces structures consiste en une imbrication de plusieurs systèmes organisationnels mutuellement incompatibles dont aucun n'est susceptible d'ordonner le texte dans sa totalité, mais dont la juxtaposition même, du fait même qu'elle se reproduit à tous les niveaux du récit (au niveau thématique, au niveau énonciatif, au niveau compositionnel, au niveau syntaxique) confère paradoxalement à ce dernier une profonde unité, qui ne saurait être décrite autrement que comme une unitas multiplex: «C'est la variation elle-même qui est systématique » (Deleuze et Guattari 1980 : 118).

Nous avons pu montrer également comment une pratique de la transgression syntaxique permet d'obtenir une « extension des possibilités de la langue » (Riffaterre $1983: 42$ ), afin de verbaliser la complexité du réel. On aboutit ainsi à une conclusion qui pourrait sembler paradoxale, voire même contre-intuitive : d'une certaine façon, la démarche scripturale de Simon correspond à une visée réaliste! Certes, il ne s'agit pas d'un réalisme comme celui qui se rencontre dans le roman du XIX ${ }^{\mathrm{e}}$ siècle, fondé sur des principes d'ordre logique, de hiérarchie, d'univocité de la signification etc. Le réalisme simonien n'est pas analytique mais synthétique, il n'est pas réductionniste mais intégrateur, il n'est pas déterministe mais s'attache au contraire à rendre compte de l'indétermination ontologique sous toutes ses formes : on est loin de la Weltanschauung d'un Zola par exemple...

Sur le plan historique, notre étude aura permis de montrer que l'œuvre simonienne se situe en fait à la croisée de deux courants littéraires : d'une part le roman moderniste "subjectiviste " (Proust, Joyce, Faulkner) et ses techniques narratives et stylistiques bien connues (focalisations internes, emploi du monologue intérieur sous toutes ses formes, mise en place d'une «écriture de la sensation » ...); d'autre part, le roman postmoderne (Cortázar, Calvino, Pynchon, Fowles, Vonnegut etc.) et ses transgressions formelles audacieuses (métalepses narratives, mise en place de «mondes possibles » irréductiblement contradictoires, hybridation généralisée des formes verbales et/ou des structures textuelles etc.). A l'évidence, l'œuvre de Simon (à tout le moins les romans des années 1960-70) est bien plus proche du second courant que du premier.

Mais notre démonstration a surtout une portée métathéorique : nous espérons avoir montré la nécessité absolue d'une prise en compte rigoureuse du fait linguistique dans l'approche du texte littéraire. Simon est un auteur au style immédiatement reconnaissable, chez qui syntaxe et vision du monde sont inextricablement liées. L'analyse de son travail ne peut donc raisonnablement faire l'économie d'une approche linguistique serrée. Pourtant, même si les choses paraissent changer, force est de constater la relative timidité des approches directement linguistiques et stylistiques. Quelles sont les causes de cette timidité ? Il y en a au moins deux. Cette partie de la recherche est freinée par un cloisonnement institutionnalisé entre les disciplines (littérature d'un côté, langue de l'autre) qui ne favorise pas les bienfaits féconds que l'une pourrait apporter à l'autre. Il faut également prendre en considération le conflit historique entre approches phénoménologiques et approches textualistes de l'œuvre de Simon, qui 
a grevé les études simoniennes pendant une vingtaine d'années: en rejetant massivement le structuralisme autoréflexif d'inspiration ricardolienne, la critique phénoménologique des années 1980 et '90 a aussi répudié tout ce qui ressemblait de près ou de loin à une approche « formaliste », jetant ainsi le bébé avec l'eau du bain... Entre formalisme «à l'ancienne » et thématisme d'inspiration phénoménologique, une approche authentiquement stylistique peine encore à trouver sa place, ce à quoi nous avons tenté ici de remédier.

\section{Références bibliographiques}

Benveniste, É. (1966). Problèmes de linguistique générale. Paris : Gallimard, coll. « Tel », t. 1.

Berrendonner, A. et Reichler-Béguelin, M.-J. (1989). «Décalages : les niveaux de l'analyse linguistique ». Langue française, 81, pp. 99-125.

Berrendonner, A. (1990). « Pour une macro-syntaxe ». Travaux de linguistique, Gand : Duculot, 21, pp. 25-36. - (2002). «Les deux syntaxes ». Verbum, XXIV, 1-2, pp. 23-36.

Bikialo, S. (2000). « Les virgules de Claude Simon ». La Licorne, 52, pp. 217-229.

Blanche-Benveniste, C. et Jeanjean C. (1987). Le Français parlé : transcription et édition. Paris : Didier, coll. "Publications du trésor général des langues et parlers français ».

Calvino, I. (2003). «Le Défi au labyrinthe ». In Défis aux labyrinthes. Textes et lectures critiques, tome 1, trad. de l'italien par Jean-Paul Manganaro et Michel Orcel. Paris : Seuil, pp. 101-116.

Cohn, D. (1981). La Transparence intérieure. Modes de représentation de la vie psychique dans le roman, trad. de l'américain par Alain Bony. Paris : Seuil, coll. «Poétique ».

Connor, S. (2004). «Postmodernism and literature». In The Cambridge companion to postmodernism, Steven Connor dir. Cambridge : Cambridge University Press, pp. 62-81.

Delas, D. et Filliolet, J. (1973). Linguistique et poétique. Paris : Larousse.

Deleuze, G. (1968). Différence et répétition. Paris : PUF, coll. «Épiméthée ».

Deleuze, G. et Guattari, F. (1980). Capitalisme et schizophrénie, vol. 2, Mille plateaux. Paris : Minuit, coll. «Critique ».

Duffy, J. H. (2006). «La Bataille de Pharsale : notice». In Claude Simon, Euvres, tome I. Paris : Gallimard, coll. «Bibliothèque de la Pléiade », pp. 1379-1403.

Faure, É. (1976 [ $\left.\left.{ }^{1} 1914\right]\right)$. Histoire de l'art. L'art renaissant. Paris : Gallimard, coll. « Folio Essais ».

Fokkema, D. W. et Calinescu, M. dirs (1987). Exploring postmodernism. Amsterdam/Philadelphie : John Benjamins.

Fokkema, D. W. et Bertens, H. dirs (1997). A comparative history of literatures in European languages, vol. XI, International postmodernism. Theory and literary practice. Amserdam/Philadelphie : John Benjamins.

Fokkema, D. W. (1997). « The semiotics of literary postmodernism ». In Fokkema et Bertens dirs 1997 : 15-42.

Hassan, I. (1982 [1971]). The Dismemberment of Orpheus : toward a postmodern literature. Madison : The University of Wisconsin Press.

- $\quad$ (1987). «Pluralism in postmodern perspective ». In Fokkema et Calinescu dirs 1987 : 17-39.

Hutcheon, L. (1988). A Poetics of postmodernism. New York/Londres : Routledge.

Jenny, L. (1997). « Sur le style littéraire ». Littérature, 108, pp. 92-101.

- (2000). «Du style comme pratique ». Littérature, 118, pp. 98-117.

Labov, W. (1976). Sociolinguistique, trad. de l'américain par Alain Kihm. Paris : Minuit, coll. « Le Sens commun ».

Mazaleyrat, J. et Molinié, G. (1989). Vocabulaire de la stylistique. Paris : PUF.

McHale, B. (1987). Postmodernist fiction. New York/Londres : Methuen.

Neveu, F. (1998). « Macrosyntaxe. Le problème des niveaux de l'analyse syntaxique dans La Route des Flandres ». L'information grammaticale, 76, pp. 38-41. 
Piat, J. (2005). «Conscience phrastique et faits de prédication : l'illisible linguistique dans le Nouveau Roman ». In Claire Badiou-Monferran et al. dirs, La Langue, le style, le sens. Études offertes à Anne-Marie Garagnon. Paris : L'Improviste, coll. « Les aéronautes de l'esprit», pp. 203-214.

Proust, M. (1987). À la recherche du temps perdu. Paris : Gallimard, coll. « Bibliothèque de la Pléiade », tome I.

- (1988a) : A la recherche du temps perdu. Paris : Gallimard, coll. « Bibliothèque de la Pléiade », tome II.

- (1988b) : À la recherche du temps perdu. Paris : Gallimard, coll. «Bibliothèque de la Pléiade », tome III.

- (1989) : À la recherche du temps perdu. Paris : Gallimard, coll. «Bibliothèque de la Pléiade », tome IV.

Rannoux, C. (1997). L'Écriture du labyrinthe: Claude Simon, La Route des Flandres. Orléans : Paradigme, coll. «Références ».

Ricardou, J. (1971). Pour une théorie du nouveau roman. Paris : Seuil, coll. « Tel Quel» .

Ricardou, J. et Van Rossum-Guyon, F. dirs (1972). Nouveau Roman: hier, aujourd'hui. Paris : Union Générale d'Éditeurs, t. II.

Riffaterre, M. (1971). Essais de stylistique structurale, trad. de l'américain par Daniel Delas. Paris : Flammarion, coll. " Nouvelle Bibiliothèque Scientifique ».

- (1983). Sémiotique de la poésie. Paris : Seuil, coll. «Poétique ».

Sarkonak, R. (1986). Claude Simon : les carrefours du texte. Toronto : Paratexte.

Schaeffer, J.-M. (1996). Les Célibataires de l'art. Pour une esthétique sans mythes. Paris : Gallimard, coll. « NRF Essais ».

Simon, C. (1962). «Entretien. Claude Simon parle », entretien avec Madeleine Chapsal. In L'Express, 564, 5 avril 1962 , pp. 32-33.

- (1972). «La fiction mot à mot» In Ricardou et Van Rossum-Guyon dirs 1972: 73-97; suivi d'une discussion, pp. 99-116.

(1977). «Un Homme traversé par le travail», entretien avec Alain Poirson et Jean-Paul Goux. In $L a$ Nouvelle Critique, 105, juillet 1977, pp. 32-44.

Sykes, S. (1979). Les Romans de Claude Simon. Paris : Minuit, coll. «Critique ».

Vouilloux, B. (2005). «Les prédicats stylistiques ». In De la langue au style, Jean-Michel Gouvard dir. Lyon : PUL, coll. « Textes et langue », pp. 319-355.

Yocaris, I. (2002). L'Impossible totalité. Une étude de la complexité dans l'œuvre de Claude Simon. Toronto: Paratexte.

- (2006a). «La discohérence dans Triptyque et Leçon de choses ». In Cohérence et discours, Frédéric Calas dir. Paris : PUPS, coll. « Travaux de stylistique et de linguistique françaises : études linguistiques », pp. 399-408.

(2006b). «Une poétique de l'indétermination: style et syntaxe dans La Route des Flandres ». Poétique, 146, pp. 217-235.

_ (2008). «Style et référence : le concept goodmanien d'exemplification ». Poétique, 154, pp. 225-248.

Zemmour, D. (1998). «Le participe présent dans La Route des Flandres : écriture du souvenir et quête de l'instant ». L'Information Grammaticale, 76, pp. 42-45.

- (2008). Une syntaxe du sensible : Claude Simon et l'écriture de la perception. Paris : PUPS, coll. «Travaux de stylistique et de linguistique françaises / Bibliothèque des styles ».

\footnotetext{
${ }^{1}$ Ainsi par exemple « Bataille » fait référence à la bataille de Pharsale, aux diverses représentations de batailles que le protagoniste du roman admire dans les tableaux italiens du quattrocento et du cinquecento, mais aussi au corps-àcorps de sa maîtresse avec son amant, sans oublier le derby local de football auquel il assiste près de Pharsale, en Thessalie. «Guerrier» renvoie - entre autres - à un soldat ivre enfermé en prison, au rival du protagoniste qui " prend d'assaut » sa maîtresse, aux guerriers des tableaux italiens de la Renaissance, à un étudiant ivre brandissant une épée en carton lors d'un bal masqué et au boxeur représenté dans une bande dessinée, lui aussi apparemment trahi par une femme. «César » évoque à la fois Jules César contemplant le champ de la bataille de Pharsale, un lieudit situé justement près de la bourgade de Pharsale et appelé Kaissares («les Césars»), des billets de banque à l'effigie de César etc.
} 
${ }^{2} \mathrm{Cf}$. BP, 20 : «modèle petite garce qui le trompait avec tout le monde Ce pauvre Charles avec les femmes il était d'une naïveté et celle-là pour enlever sa culotte il ne lui fallait». On note évidemment ici la présence du verbe «tromper», qui permet effectivement de relier cette phrase au texte (2) par le biais d'une discrète réactivation étymologique ( « tromper » est apparemment un dérivé de « trompe »).

3 On voudra bien noter que ce flottement indéterministe entre plusieurs systèmes de règles différents qui s'interpénètrent constitue un des traits distinctifs de base de l'espace rhizomatique : Deleuze et Guattari (1980 : 4748) expliquent ainsi que, au sein de cet espace, on voit justement coexister des «multiplicités arborescentes » (en l'occurrence, les agencements syntaxiques linéaires et hiérarchisés hérités du roman «traditionnel ») et des « multiplicités rhizomatiques » (en l'occurrence, les agencements syntaxiques complexes et «acentrés » mis en place dans La Bataille de Pharsale).

${ }^{4}$ Pour plus de précisions sur ce point, cf. Yocaris 2006b.

${ }^{5}$ En témoigne notamment une citation approximative du Temps retrouvé qui revient à deux reprises dans la première partie de La Bataille de Pharsale: "édicules Rambuteau s'appelaient des pistières Sans doute dans son enfance n'avait-il pas entendu l'o et cela lui était resté Il prononçait donc ce mot incorrectement mais ... » (BP, 22 ; italiques du texte ; cf. BP, 38 ; v. Proust 1989 : 329 : «Il [le maître du Grand Hôtel de Balbec] croyait que ce que M. de Rambuteau avait été si froissé un jour d'entendre appeler par le duc de Guermantes des "édicules Rambuteau" s'appelait des pistières. Sans doute dans son enfance n'avait-il pas entendu l'o, et cela lui était resté. Il prononçait donc ce mot incorrectement mais perpétuellement. »; cf. Proust 1988b : 694-695). Dans cet extrait, la lettre «o » renvoie à un espace vide pour deux raisons: (a) parce qu'elle n'est pas prononcée par le personnage ; (b) parce qu'elle figure justement le trou d'un urinoir ...

${ }^{6}$ Sur le concept de «monologue remémoratif », v. Cohn $1981: 210$.

${ }^{7}$ Sur le concept de «clause », cf. Berrendonner et Reichler-Béguelin 1989, Berrendonner 1990, 2002, Neveu 1998. Sur le recours à ce concept pour rendre compte des spécificités inhérentes à la syntaxe simonienne, cf. Zemmour 2008 .

${ }^{8}$ Simon, qui s'était porté volontaire en 1936 pour se battre aux côtés des militants du POUM à Barcelone, n'a jamais fait mystère de sa fascination pour les anarchistes qui faisaient partie du mouvement et leur modus operandi : v. p. ex. Simon $1962: 32$

${ }^{9}$ Le concept est emprunté à William Labov, dont les travaux sont cités et commentés dans le quatrième « chapitre » de Mille plateaux, «Postulats de la linguistique » (cf. Deleuze et Guattari 1980 : 118-123, 131, Labov 1976).

${ }^{10}$ Sur les concepts de macrocontexte et de norme macrocontextuelle, v. Riffaterre 1971 : 80-94.

${ }^{11}$ Sur le concept d'exemplification et ses possibles applications dans le domaine de la stylistique, cf. Schaeffer 1996 : 308-332, Jenny 1997, 2000, Vouilloux 2005, Yocaris 2008.

${ }^{12}$ D'où les remarques de Julien Piat sur « la rencontre problématique entre deux programmes de lecture concurrents : un programme syntaxique et un programme sémantico-logique» (Piat $2005: 211$ ) dans les textes de Simon, de Beckett ou de Pinget.

${ }^{13}$ Cf. Deleuze et Guattari 1980 : 20 et passim.

${ }^{14}$ Ibid.

${ }^{15}$ D'où l'importance décisive accordée dans la plupart des ouvrages postmodernes à un procédé comme la mise en abyme : en termes calviniens, celle-ci donne à voir effectivement « la carte la plus détaillée possible du labyrinthe » (Calvino 2003 : 115) dans lequel le lecteur/les personnages de La Route des Flandres, de Marelle, du Pendule de Foucault, de La Maison de rendez-vous, de L'Emploi du temps, du Château des destins croisés ou du Jardin aux sentiers qui bifurquent se trouvent (in)volontairement égarés. 